Chin, B. \& Knight, S. G. (1957). J. gen. Microbiol. 16, 642-646

\title{
Growth of Trichophyton mentagrophytes and Tricho- phyton rubrum in Increased Carbon Dioxide Tensions
}

\author{
By B. CHIN AND S. G. KNIGHT \\ Department of Bacteriology, University of Wisconsin, \\ Madison, Wisconsin, U.S.A.
}

SUMMARY: The formation of macrospores in normally mycelial strains of Trichophyton mentagrophytes and T. rubrum was induced by growing the cultures in a gas phase containing air $+12-24 \%(\mathrm{v} / \mathrm{v}) \mathrm{CO}_{2}$. When conidial transfers were made from strains which had thus been induced to form macrospores and were incubated in normal air, the subsequent growth was mycelial and downy. One strain of each fungus, normally conidial and granular, formed arthrospores under increased $\mathrm{CO}_{2}$.

The bases for genus and species differentiation of the superficial dermatophytes are shape and size of macroconidia and gross cultural characteristics (Emmons, 1934). Identification of these Fungi imperfecti is complicated by loss of the ability to form macroconidia and variation in cultural morphology; most of the dermatophytes exist in two morphological types, conidial and mycelial (Wilhelm, 1947). When these fungi are first isolated from lesions on to Sabouraud's glucose agar, they usually grow as the conidial, macroconidiaforming, type. Upon repeated transfer, however, they often lose the ability to sporulate and continue growth as the mycelial type. Reversion of the mycelial to the conidial form is very uncommon. Investigations in this laboratory have shown that non-sporulating strains of Trichophyton mentagrophytes and T. rubrum can be induced to form macroconidia, thus reverting to the conidial type, by incubation in a gas phase containing concentrations of carbon dioxide above that of normal air $(c .0 .03 \%, v / v)$.

\section{METHODS}

Cultures. The cultures employed in this study included 5 strains of Trichophyton mentagrophytes (408, My-874-55, A-538, X-15, X-10) and 6 strains of T. rubrum (T-3, X-14, My-38-56, X-2, My-274-56, My-640-55). Of these, only strains X-10 and X-14 were the conidial type and formed typical macroconidia and microconidia on Sabouraud's glucose agar when incubated in normal air; the other strains formed microconidia but no macronidia under these conditions. Strain 408 formed vegetative mycelium without differentiated structures. All cultures were maintained as stocks on slopes of Sabouraud's glucose agar (Difco). After inoculation, slope cultures were incubated at $30^{\circ}$ for 1 week then sealed with tinfoil and wax stored at $5^{\circ}$ until used.

Inoculum. The inoculum was prepared by dispersing the growth from a stock slope with $10 \mathrm{ml}$. sterile water in a Waring blender for $1 \mathrm{~min}$. One drop of this suspension was inoculated on to the centre of each of a series of 
Sabouraud's glucose agar plates. Eight or more of these plates were incubated in each concentration of carbon dioxide.

$\mathrm{CO}_{2}$ concentration. Carbon dioxide concentration was adjusted, after a set of plates had been sealed in a Brewer's anaerobic jar, by drawing a vacuum of approximately $30 \mathrm{~mm}$. $\mathrm{Hg}$ in excess of the desired concentration of $\mathrm{CO}_{2}$. The desired amount of $\mathrm{CO}_{2}$ was run in from a cylinder and was measured by noting the fall in negative pressure on a manometer, then the valve of the jar was momentarily opened to return the internal pressure of the jar to atmospheric Control plates were incubated in laboratory air.

All cultures were incubated at $30^{\circ}$ and the $\mathrm{CO}_{2}$ concentrations were readjusted daily. During the period of incubation, 2 plates from each concentration were removed at 2- to 3-day intervals for microscopic examination of the mycelium. At the end of 7 or more days of incubation, all the remaining plates were removed and examined macroscopically and microscopically.

\section{RESULTS}

Macroconidia formation by 3 out of 4 mycelial strains of Trichophyton mentagrophytes and 5 out of 5 mycelial strains of $T$. rubrum was induced by incubation at increased concentrations of $\mathrm{CO}_{2}$; Table 1 indicates the response to $\mathrm{CO}_{2}$ of all the cultures of definite history that were tested. However, this response to $\mathrm{CO}_{2}$ was discovered with a stock strain of $T$. mentagrophytes and 2 similar strains of $\boldsymbol{T}$. rubrum which are not included in this discussion because of their unknown backgrounds. Pl. 1, fig. 1, shows typical mycelial growth of T. rubrum T-3; Pl. 1, fig. 2, shows the conidial growth of the same strain under $16 \%(\mathrm{v} / \mathrm{v}) \mathrm{CO}_{2}$; this profuse formation of macroconidia is typical of most of the other strains when incubated under $\mathrm{CO}_{2} . T$. mentagrophytes 408 , which did not respond to increased $\mathrm{CO}_{2}$, was not typical of other downy strains; it was completely vegetative at all times, producing neither macronor microconidia. The conidial strains $T$. rubrum X-14 and T. mentagrophytes X-10 formed arthrospores exclusively at $\mathrm{CO}_{2}$ concentrations of $16 \%$ or more; Pl. 1, fig. 3, shows arthrospore formation by $T$. rubrum X-14. To determine that the increased concentration of $\mathrm{CO}_{2}$ and not the decreased concentration of oxygen was, inducing macroconidia formation, $T$. mentagrophytes strain A-538 was incubated in an atmosphere containing $20 \%(\mathrm{v} / \mathrm{v})$ more nitrogen than normal. Under these conditions, macroconidia were not produced and no changes in morphology were apparent.

As macroconidia formation increased with increasing $\mathrm{CO}_{2}$ concentrations, the macroscopic appearance of mycelial strains changed gradually from downy to granular; Pl. 1, fig. 4, shows the increased granulation and pigmentation of Trichophyton rubrum T-3 as the $\mathrm{CO}_{2}$ concentration was increased. As the concentration of carbon dioxide in the atmosphere was increased, the diameters of the colonies decreased somewhat (Table 2).

At the concentrations of $\mathrm{CO}_{2}$ shown in Table 1 the dermatophytes fell into four groups with regard to pigment formation: (i) the intensity of pigment increased stepwise as $\mathrm{CO}_{2}$ concentration was increased from 0 to $24 \%$ 
Table 1. The effect of increased concentrations of carbon dioxide on macroconidia formation by strains of Trichophyton mentagrophytes and T. rubrum.

Three plates were examined for each concentration.

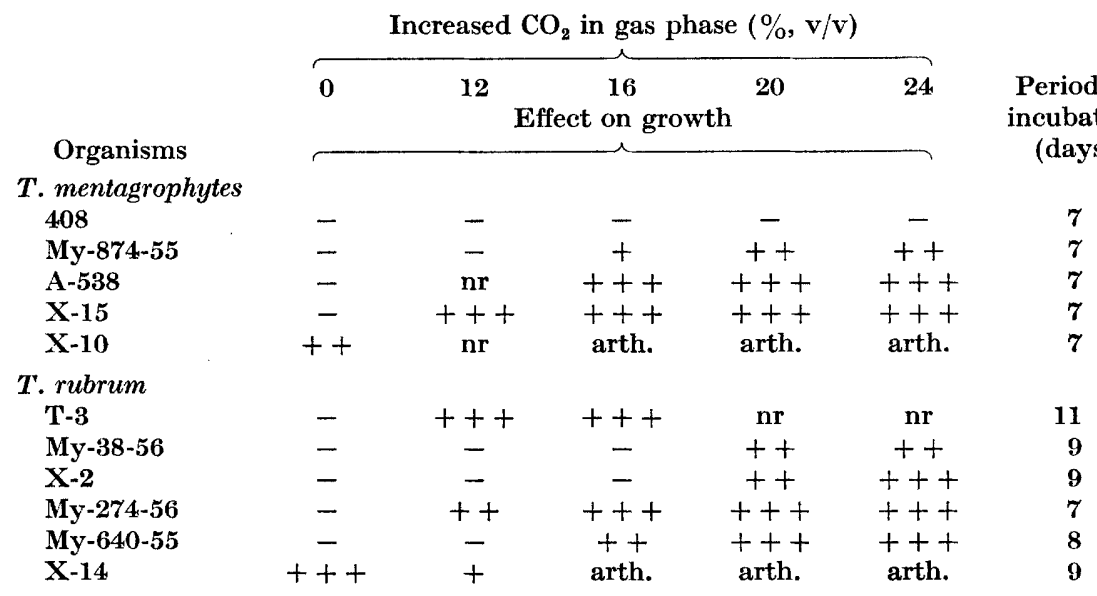

$-=$ no macroconidia; $+=$ occasional macroconidia; $++=$ abundant macroconidia; $+++=$ profuse macroconidia. arth. $=$ arthrospore formation only. $\mathrm{nr}=$ not run at this concentration.

Table 2. Effect of increased carbon dioxide concentrations on the colony diameter of Trichophyton mentagrophytes and T. rubrum

Three plates were averaged for each concentration.

\begin{tabular}{|c|c|c|c|c|c|c|}
\hline & & $\mathrm{CO}$ & pha & $v / \mathbf{v})$ & & \\
\hline & 0 & ${ }_{\mathrm{C}}^{12}$ & $\begin{array}{c}16 \\
\text { iame }\end{array}$ & $\begin{array}{l}20 \\
\text { n.) }\end{array}$ & 24 & $\begin{array}{l}\text { Period of } \\
\text { incubation }\end{array}$ \\
\hline T. mentagroph & & & & & & \\
\hline 408 & 54 & 50 & 49 & 47 & 46 & 7 \\
\hline My-874-55 & 52 & 48 & 45 & 39 & 36 & 7 \\
\hline A-538 & 40 & $\mathrm{nr}$ & 30 & 26 & 25 & 7 \\
\hline X-15 & 47 & 27 & 26 & 27 & 25 & 7 \\
\hline$X-10$ & 47 & $\mathbf{n r}$ & 34 & 32 & $3 n$ & 7 \\
\hline T. rubrum & & & & & & \\
\hline$X-14$ & 25 & 21 & 20 & 18 & 18 & 9 \\
\hline My-38-56 & 34 & 28 & 25 & 23 & 21 & 9 \\
\hline $\mathrm{X}-2$ & 35 & 33 & $\mathbf{3 0}$ & 26 & $\mathbf{2 4}$ & 9 \\
\hline My-274-56 & 25 & 23 & 18 & 17 & 16 & 7 \\
\hline
\end{tabular}

(strains T-3, My-874-55, A-538); (ii) the intensity of pigment decreased stepwise as $\mathrm{CO}_{2}$ concentration was increased from 0 to $24 \%$ (strains $408, \mathrm{My}-38-56$, $\mathrm{X}-10, \mathrm{X}-14, \mathrm{My}-640-55$ ); (iii) the intensity of pigment increased from 0 to $12 \% \mathrm{CO}_{2}$, then decreased from 12 to $24 \% \mathrm{CO}_{2}$ (strain $\mathrm{X}-15$ ); (iv) the intensity of pigment decreased from 0 to $12 \% \mathrm{CO}_{2}$, then increased from 12 to $24 \% \mathrm{CO}_{2}$ (strains My-274-56, X-2). There seemed to be no consistent relationship 
between the two species or strains within a species regarding the relation of pigmentation and $\mathrm{CO}_{2}$ concentration.

Conidial subcultures from mycelial strains which were induced to form macroconidia under increased $\mathrm{CO}_{2}$ were made at the end of the incubation periods. After incubation for a week in air at $30^{\circ}$, these subcultures again had the typical downy gross morphology and no macroconidia were found upon microscopic examination.

\section{DISCUSSION}

In this laboratory the terms 'downy' and 'granular' are used for macroscopic descriptions, and 'conidial' and 'mycelial' are used for microscopic descriptions. The difference between granular and downy is not clear-cut; one strain may appear more downy or granular than another and intermediates exist between both extremes. Conidial strains (X-10, X-14) form macroconidia on slopes of Sabouraud's glucose agar in normal atmospheric conditions of incubation; mycelial strains (My-874-55, A-538, My-38-56, X-2, My-274-56, My-640-55, X-15, T-3) do not, both conidial and mycelial strains form microconidia. A granular strain is usually of the conidial type and a downy strain is usually in the mycelial type. However, strains which appear downy may form a few macroconidia and strains which appear granular because of intensive microconidia formation may form no macroconidia. Because of this colonial variation, any cultural condition resulting in more consistent macroscopic and microscopic morphology is noteworthy.

The reason for the change from the conidial type to the mycelial type in the dermatophytes is not known, although Wilhelm (1947) and Robbins \& McVeigh (1949) suggested that a mutation at some point in the life cycle of the fungus was involved. The observations made here, namely, the rapid change from the mycelial to the conidial type by increased concentrations of carbon dioxide and the immediate return to the mycelial type upon incubation in laboratory air, seems to argue against mutation.

Whether carbon dioxide acts as a physical or chemical agent in producing these morphological changes is not at present known. Macrospore formation under increased $\mathrm{CO}_{2}$ concentration may be associated with the metabolism of purines and pyrimidines or tricarboxylic cycle components since $\mathrm{CO}_{2}$ is readily fixed into these compounds by many micro-organisms. However, it is not apparent why some strains form macroconidia readily on Sabouraud's glucose agar in normal atmospheric conditions, while others must be induced by increased concentrations of carbon dioxide to form these spores and lose the ability immediately upon transfer to normal atmosphere.

The authors thank Dr Libero Ajello of the Communicable Disease Center, Chamblee, Georgia, U.S.A., for the cultures used in this study. This work was supported by grants from the University of Wisconsin Research Foundation and the National Institutes of Health, grant number E-1201. 


\section{REFERENCES}

Emmons, C. W. (1934). Dermatophytes: natural groupings based on the form of spores and accessory organs. Arch. Derm. Syph., N.Y. 30, 337.

Robbins, W. J. \& McVeigh, I. (1949). 'The 'dual phenomenon' and Trichophyton mentagrophytes. Mycologia, 41, 128.

Wilfelm, S. (1947). The dual phenomenon in the dermatophytes. Mycologia, 39, 716.

\section{EXPLANATION OF PLATE}

Fig. 1. Mycelium of Trichophyton rubrum T-3 incubated in air; a few microconidia can be seen $(\times \mathbf{2 8 0})$.

Fig. 2. Mycelium of T. rubrum T-3 showing profuse macroconidia formation after incubation for 11 days in $\mathrm{air}+16 \%(\mathrm{v} / \mathrm{v}) \mathrm{CO}_{2}(\times 280)$.

Fig. 3. Arthrospore formation by granular $T$. mentagrophytes X-10 incubated in air $+16 \%$ $\mathrm{CO}_{2}(\times 280)$.

Fig. 4. Giant colonies of $T$. rubrum T-3 incubated 9 days at different concentrations of $\mathrm{CO}_{2}$. Increased granulation and pigment formation is apparent.

(Received 3 December 1956) 
Journal of General Microbiology, Vol. 16, No. 3
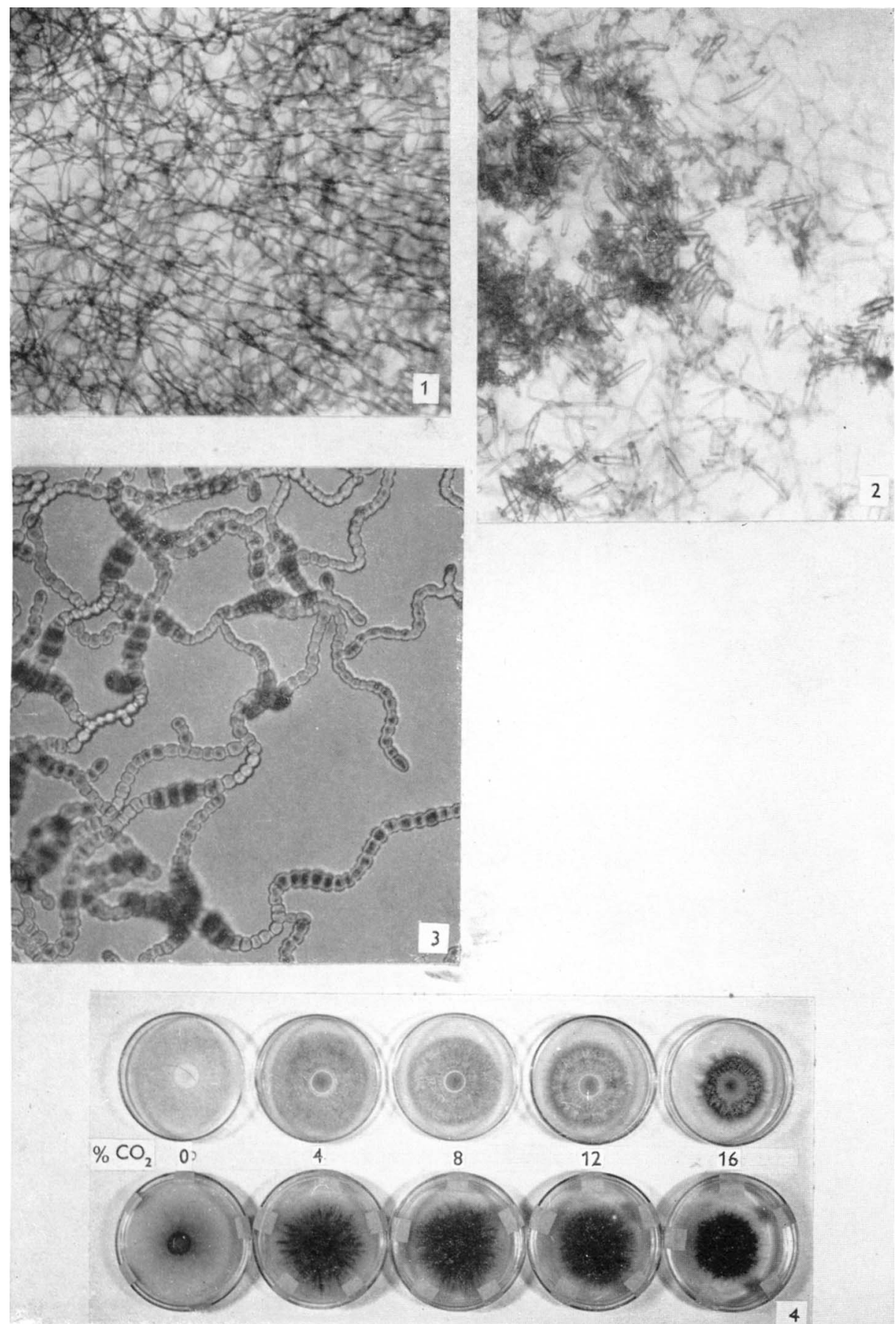

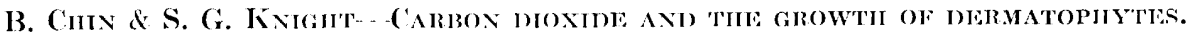
PIATE 1 